Association for Information Systems

AIS Electronic Library (AISeL)

ICEB 2001 Proceedings

International Conference on Electronic Business

(ICEB)

Winter 12-19-2001

Customized Internet News Services Based on Customer Profiles

Ting-Peng Liang

Hung-Jen Lai

Follow this and additional works at: https://aisel.aisnet.org/iceb2001

This material is brought to you by the International Conference on Electronic Business (ICEB) at AIS Electronic Library (AISeL). It has been accepted for inclusion in ICEB 2001 Proceedings by an authorized administrator of AIS Electronic Library (AISeL). For more information, please contact elibrary@aisnet.org. 


\title{
Customized Internet News Services Based on Customer Profiles
}

\author{
Ting-Peng Liang anf Hung-Jen Lai \\ Department of Information Management \\ National Sun Yat-sen University \\ Kaohsiung, Taiwan \\ Email: tpliang@mail.nsysu.edu.tw
}

\section{(Extended Abstract)}

\begin{abstract}
The rapid propagation of the Internet and information technologies has changed the nature of many industries through easy collection, analysis, and sharing of information. Rapid response and product customization are major concerns in their marketing and strategic planning. This is particularly significant in the industries that offer digital contents to their clients, such as consulting and news services. In this paper, a news recommendation system that allows a news service provider to analyze its customer profile and then produce customized news services is developed. An empirical study using actual news provided by the China Times shows that the recommendation system outperforms the traditional headline news compiled by the news editor in both objective performance indices and customer satisfaction.
\end{abstract}

\section{Introduction}

The rapid propagation of the Internet, along with the evolution of information technologies, has changed the nature of many businesses. The large amount of transactional data collected from the use of information systems allows a company to better understand customer needs and to integrate the knowledge into their product design and marketing plans. For physical products (e.g., computers and televisions), mass customization and fast response to market needs become critical to remaining competitive. For digital products (e.g., news services and other Internet content providers, ICP), personalized services that offer tailored content to different clients based on their interests become feasible and necessary.

In fact, the Internet provides an excellent platform for news or other content to be delivered. Traditionally, the editor of a newspaper determines the relative importance of reports and put the ones with high interests into a category called headlines. All readers view the same set of headline news, no matter whether they are in hard copy or in the web page. This certainly is not the best way to serve clients with diverse interests. Early in 1992, Lorrie identified three major advantages of e-newspapers that offer news services on websites or through email delivery: (1) timely, (2) richness, and (3) customization. The ability to customize services is what a reader needs.

In order to produce personalized services, the news provider has to know the preference of different customers and make proper recommendations. The system that tailors news to fit the needs of different readers is called a news filtering or news recommendation system. Several approaches have been proposed to provide personal news services. However, most of them require either tedious news classification or the customer' s rating after reading, which is very inconvenient (e.g., Mock and Vemuri, 1997; Sakagami and Kamba, 1997). The existing methods also have difficulties in capturing the dynamics of customer interests.

In this paper, we propose a new approach that builds customer profiles from their browsing behavior and analyzes these profiles to produce personal news services delivered on the web.

\section{Literature Review}

A few filtering mechanisms have been proposed in the past. A typical one is to ask the reader to report his interest after reading. The system can then build a profile of the reader and make recommendation accordingly. For example, Mock and Vemuri [4] presented the Intelligent News Filtering Organization System (INFOS) that asked each reader to indicate whether he liked the report. The system reorganizes the order of news based on the revealed preference. Results from a pilot test show that INFOS can effectively reduce the reader's search load.

Another approach is behavior-based. For instance, Sakagami and Kamba [6] developed the ANATAGONOMY that learns reading preference from the browsing behavior (e.g., scroll, enlarge windows, etc.) of a user. The system has a learning engine and a scoring engine to produce personalized web news.

News filtering and recommendation can also be performed based on feedback from others. For instance, Konstan, et al. [3] proposed a system, called GroupLens, which summarized the feedback from previous readers to allow the next reader to determine whether to read it. This is called collaborative filtering. A system proposed by Balabanovic and Shoham [1] combines content analysis and collaborative filtering. It takes into account the association between a reader and the theme of a report to identify the discrepancy between different individuals.

\section{The Personal News Recommendation Mechanism}

In this section, we propose a new approach that is based on content structure and browsing behavior. In particular, the approach focuses on the time a user spent on reading an article. We shall call it a time-based behavior analysis for user profile. The basic assumption of the approach is that a reader will spend a reasonable amount of time to read if the article is of interest. Reading too long or too short is unusual. 
The approach consists of four major modules: structure analysis, reader profile analysis, rating for recommendation, and learning .

\subsection{Structure Analysis}

The first step for personalized service is to analyze the structure of the news. The foundation of structure analysis is to identify keywords in a report and to build the keyword dictionary. Keywords in each report are identified based on the property of the words. In our mechanism, we use nouns with a particular emphasis on the role and issues mentioned in the report.

After identifying keywords, we further analyze the position and frequency of keywords. Major steps include: (1) Determine whether synonyms exist, such as American Online $=$ AOL, President Clinton $=$ Bill Clinton, Yahoo $=$ YHOO, etc.. (2) Calculate the frequency of keywords, (3) Adjusting the weight of each words by their position in the article, (4) Eliminate noises (i.e., words whose frequencies are lower than a hurdle), (5) Converting all keyword frequencies into a ratio, which is the frequency of a keyword/sum(frequencies of all keywords). The structure of the report is the collection of valid keywords along with their respective frequency ratio, such as [Inside.com (.31), Yahoo (.28), portal (.21), web (.20)].

\subsection{Analysis of Reader Profile}

Analysis of customer profile is based on the browsing behavior, particularly the time spent in reading a report. The computer records the beginning and ending time that a user reads a report. The data are then analyzed in the following steps to build the reading profile: (1) Calculate the average reading speed of the user, (2) Calculate the interest level of a user on the report: The interest level of a report is calculated by the time spent in reading it and is represented by the ratio of dividing the actual reading time by the estimated reading time, where the estimated reading time $=$ total words in the report/average reading speed. (3) Conduct recency adjustment: recent reports are given higher weights, (4) Calculate the adjusted interest level of a reader on a report is the product of the interest level from step ) multiplied by the recency weight result from step 4, (5) Build the customer profile: The interest profile of a user is to multiply news structure by the interest level.

\subsection{Rating and Recommendation}

Rating and recommendation determine the matching between a news report and a reader. If the matching level is higher than the threshold value, the report will be recommended to the reader. Otherwise, it is dropped. Steps for matching reports with a reader include: (1) Determine the structure of the report, (2) Calculate the matching level: The matching level is calculated by aggregating the interest levels of different keywords, and (3) Recommend news based on matching levels: A hurdle can be set to screen out reports with low matching levels. The reports whose matching levels are higher than the threshold value will be recommended.

\subsection{Learning}

In the real application, the readers' interests can be very dynamic. Therefore, the system needs to have a learning module that compares the recommended reports and the reports that the user actually read. If a significant discrepancy is found, the system must adjust its recommendation mechanism.

\section{Empirical Study}

In order to evaluation the news recommendation mechanism, an experimental study was performed. The benchmarks were the regularheadline approach (HLA) and the self-reported interests (SRI) approach. Prototype systems that present news by HLA, SRI, and browsing behavior analysis (BBA) approach were developed for the experiment.

\subsection{Experimental variables and hypotheses}

The purpose of the experiment is to compare the performance of different news presentation mechanisms. Therefore, the independent variable is news recommendation mechanisms, which include BBA, SRI, and the traditional HLA. The dependent variable is the evaluation of the systems, which include objective performance measures and subjective user satisfaction. The null hypotheses are:

H1: BBA and SRI perform equally well with HLA,

H2: BBA performs equally well with SRI.

\subsection{Experimental Systems}

Three experimental websites that provide news services were designed. The HLA system copies the regular headline news approach. That is, the home page outlines the titles of the headline news that were determined by the editor. The design of the SRI and BBA systems is the same except that their home pages are customized for each individual reader. When a user logs into the SRI or BBA system, the computer identifies his reading interests from the user profile and then composes the recommended news into the homepage.

All systems have automated recording module that keeps track of the following data: (1) which news the subject click to read, (2) time spent in reading a particular news, (3) time spent in choosing a news to read (i.e., the time that the subject logs on the system but not for reading the news).

\subsection{Measurement Instruments}

The background questionnaire collects data about the subject. It includes 11 questions: (1) most interesting category of news (a total of 13 categories), (2) least interesting category of news, (3) if you have 20 minutes, would you browse titles or read news in a news website, (4) frequency of using news websites in a week, (5) average time spent on a news website in a visit, (6) which is your most common news source (print media, TV, or web), (7) experiences in using the web, (8) motivation for using news websites, (9) importance of receiving news and information to you, (10) preference in filtering news by the system, (11) choosing news by interests or location of the title.

Both objective performance and user satisfaction of the subjects were measured. Two indices are common for 
measuring objective performance: precision and recall (Saracevic and Kantor, 1998). Precision measures the portion of recommended news that is relevant (i.e., read by the subject) and recall measures the portion of relevant news that is recommended. To explore further detail of the performance, five indices have been developed for the experiment: acceptance rate, hit rate by the number of news, hit rate by the reading time, effective usage rate, and effective reading rate. The definition of these indices is summarized in Table 2.

Table 2. Definition of Subjective Performance Measures

\begin{tabular}{|l|l|}
\hline Measure & Definition \\
\hline $\begin{array}{l}\text { Acceptance Rate } \\
\text { (AR) }\end{array}$ & $\begin{array}{l}\text { No. of recommended and read/No. of } \\
\text { recommended }\end{array}$ \\
\hline $\begin{array}{l}\text { Number Hit Rate } \\
\text { (NHR) }\end{array}$ & $\begin{array}{l}\text { No. of recommended and read/Total } \\
\text { No. of read }\end{array}$ \\
\hline Time Hit Rate (THR) & $\begin{array}{l}\text { Total time reading recommended } \\
\text { news/Total reading time }\end{array}$ \\
\hline $\begin{array}{l}\text { Effective use rate } \\
\text { (EUR) }\end{array}$ & $\begin{array}{l}\text { Total time of selecting and reading } \\
\text { news/Total time }\end{array}$ \\
\hline $\begin{array}{l}\text { Effective reading rate } \\
\text { (ERR) }\end{array}$ & $\begin{array}{l}\text { Total time of reading/Total time of } \\
\text { selecting and reading }\end{array}$ \\
\hline
\end{tabular}

The instrument for measuring user satisfaction includes four dimensions: information content, customized services, user interface, and system value. Satisfaction on information content is measured by three questions adapted from Doll [2]. Satisfaction on customized services is measured by three questions adapted from the personalized service portion of SERVQUAL[5]. Satisfaction on user interface is measured by four questions adapted from Doll[2]. Finally, a question is designed to assess the overall satisfaction of the user on the system. All questions are on a 7-point Likert scale with 1 being least agreed and 7 being most agreed. The reliability test of the questionnaire using Cronbach's alpha shows that the instrument is generally acceptable because most alpha values are higher than 0.6 .

\subsection{Experimental Design and Procedures}

The subjects were asked to participate in the experiment for four days. A total of 96 volunteered subjects were recruited at the beginning. They were divided into two groups, one of which viewed HLA and SRI (Group I) and the other viewed HLA and BBA (Group II). Nine of them dropped out during the experiment, So, we had a total of 87 effective subjects, with 43 in Group I and 44 in Group II.

Subjects in both groups were asked to view HLA in the first three days and fill out a satisfaction questionnaire after the second day. On the fourth day, subjects in Group I viewed SRI and those in Group II viewed BBA. They all filled out questionnaires again to indicate their satisfaction with the experimental system.

In order to be close to the real world, the news adopted for the experiment was actual news provided by China Times (www.chinatimes.com.tw). During the experimental period (June 7 - June 10, 2000), all news available on the website of China Times before 9:00 am were downloaded to the experimental system, organized based on different approaches, and then presented to the experimental subjects.

\subsection{Experimental Results}

During the experiment, the HLA system presents 41 news titles to all subjects in the homepage, whereas SRI and BBA present an average of 17.77 and 17.61 recommended news titles in the homepage to each subject, respectively. That is, the recommendation systems are more selective than the standard headline news version.

\section{H1: SRI and BBA perform equally well with HLA}

The results of the paired t-test indicate that SRI and BBA both outperform HLA significantly in all objective indices and user satisfaction. Therefore, we can safely conclude that the recommendation systems outperform the traditional headline approach. The null hypothesis is rejected.

H2: SRI and BBA perform equally well

BBA performs better than SRI in the rate of effective use (EUR), but worse than SRI in customization and perceived value. The overall satisfaction shows no significant difference. Therefore, the null hypothesis cannot be denied. We conclude that both recommendation mechanisms are equally good.

\section{Concluding Remarks}

In this paper, a novel approach that integrates content structure and browsing behavior analysis to customize news services is presented. The empirical evidence indicates that it performs better than the traditional headline news approach.

\section{References}

[1] Balabanovic, M. and Shoham, Y., "Fab: Content-based Collaborative Recommendataion," Communications of the ACM, 40:3, 1997, pp. 66-72.

[2] Doll, W.J. and Torkzadeh, G., "The Measurement of End-user Computing Satisfaction," MIS Quarterly, 12:2, 1988, pp. 913-923.

[3] Konstan, J. A., Miller, B. N., Maltz, D., Herlocker, J. L., Gordon, L. R., and Riedl, J., "GroupLens: Applying Collaborative Filtering to Usenet News," Communications of the ACM, Vol. 40, No. 3, Mar 1997, pp. 77-87.

[4] Mock and Vemuri, "Information Filtering Via Hill Climbing, Wordnet, and Index Patterns," Information Processing \& Management, Vol. 33, No. 5, 1997, pp. 633-644.

[5] Parasuraman, A., Zeithaml, V.A. and Berry, L.L., "SERVQUAL: A Multiple-item Scale for Measuring Consumer Perceptions of Service Quality," Journal of Retailing, 64:1, 1988, pp. 12-40.

[6] Sakagami, H. and Kamba, T., "Learning Personal Preferences on Online Newspaper Articles from User Behaviors," Computer Networks and ISDN Systems, Vol. 29, 1997, pp.1447-1455. 